\title{
Estudio Estadístico de Lesiones Premalignas de Cuello
}

\author{
HOSPITAL SAN VICENTE Y SEGURO SOCIAL - MEDELLIN
}

\author{
Dr. Jaime Uribe Duque* \\ Dr. René Díaz Correa** \\ Dr. Leonidas Rodríguez Rodríguez***
}

\section{INTRODUCGION:}

El carcinoma y las lesiones pre-malignas del cuello uterino requieren la máxima atención en un servicio de Oncología Ginecológica. Desafortunadamente, en nuestro medio ha sido casi imposible el seguimiento adecuado de las pacientes por diferentes causas y por lo tanto las estadísticas no permitían una evaluación correcta de los diferentes métodos de tratamiento, en relación con la respuesta obtenida. Gracias a la colaboración del Departamento de Sistemas y Cómputos de la Universidad de Antioquia y de destacados profesores de la Escuela Nacional de Salud Pública hemos logrado la evaluación obtenida en 2.666 pacientes con patología del cuello uterino estudiadas en la Clínica Oncológica del Hospital V. de P. e I.C.S.S. de Medellín.

\section{OBJETIVO GENERAL:}

Es el de realizar un estudio que permita ver las características, frecuencia y distribución de las lesiones del cuello uterino en las pacientes que durante el período comprendido entre los años 1970 y Octubre de 1977 consultaron al H.U.S.V. de P. e I.C.S.S. de Medellín.

*Profesor Titular de la Cátedra de Oncología Ginecológica, U. de A.

**Profesor Cátedra de Oncología Ginecológica, U. de A.

***Profesor de la Cátedra de Oncología Ginecológica, U. de A.

- Trabajo presentado en el XII Congreso Colombiano de Obstetricia y Ginecologia de Medellín Diciembre de 1.977.

\section{OBJETIVOS ESPECIFICOS :}

1 -Establecer el número de pacientes que presentaron lesiones de cuello uterino durante el período de estudio y en relación con la edad.

2. Determinar la frecuencia de las lesiones de cuello uterino de acuerdo con la edad de la primera relación sexual.

3. Informar sobre los diferentes tratamientos utilizados de acuerdo con el estado clínico.

4. Presentar los tratamientos paliativos quirúrgicos complementarios a la terapia inicial.

5. Demostrar la frecuencia porcentual de las lesiones pre-malignas y malignas con las lesiones pre-malignas y malignas relacionadas con el estado clínico.

6. Indicar la edad promedio de las pacientes de acuerdo con el estado clínico.

7. Establecer la relación que existe entre el informe anatomo-patológico de la biopsia y el material quirúrgico.

8. De acuerdo con los estados clínicos de las lesiones invasoras, carcinoma in situ y displasia, informar cuales fueron las entidades que enviaron las pacientes.

9. Demostrar a las entidades de Salud Pública, mediante estadísticas locales, el problema actual del carcinoma avanzado del cuello uterino y en ésta forma motivarlas para una financiación adecuada con miras a un diagnóstico precoz y un tratamiento oportuno.

\section{METODOLOGIA Y PROCEDIMIENTO}

Para cumplir los objetivos propuestos se hi- 
zo una revisión completa de todas las historias de las pacientes que habían consultado durante los años 1970 - 1973 y se inició el seguimiento después de codificar los datos en un formulario establecido para las pacientes nuevas. Se trata de un estudio en dos etapas: una retrospectiva y otra, prospectiva.

Se diseñó un programa para ser procesado por computador, con diferentes variables que permitieran establecer estadísticas propias sobre el comportamiento de éstas lesiones en nuestras pacientes. (ver anexos 1 y 2 ).

Se hizo un instructivo para que los formularios fueran elaborados con los mismos criterios. La evaluación de las pacientes y su clasificación fueron realizadas por el mismo equipo humano.

\section{UNIVERSO :}

Está constituído por pacientes que consul-

CUADRO No. 1 taron a la Clínica de Oncología Ginecolo. gía del H.U.S.V. de P. e I.C.S.S. de Medellín. El número fué de 2.666 pacientes que presentaban displasias, carcinoma in situ e invasor del cuello uterino.

\section{TABULACION}

La información fué tabulada en el Departamento de Sistemas y Cómputos de la Universidad de Antioquia y se presenta en cuadros gráficos.

\section{ANALISIS :}

Cuadro No. 1. Es de observar que mientras en las displasias el 50o/o de los casos comprendidos entre $Q$ y $Q$ es la edad entre 25 a 45 años. El Carcinoma in situ e invasor están comprendidos entre 35 y 55 años y el total corresponde a la edad comprendida entre 30 y 55 años. Es importante puntualizar que entre 55 y 99 años se presentaron 511 casos pero el período que comprende es de 44 años.

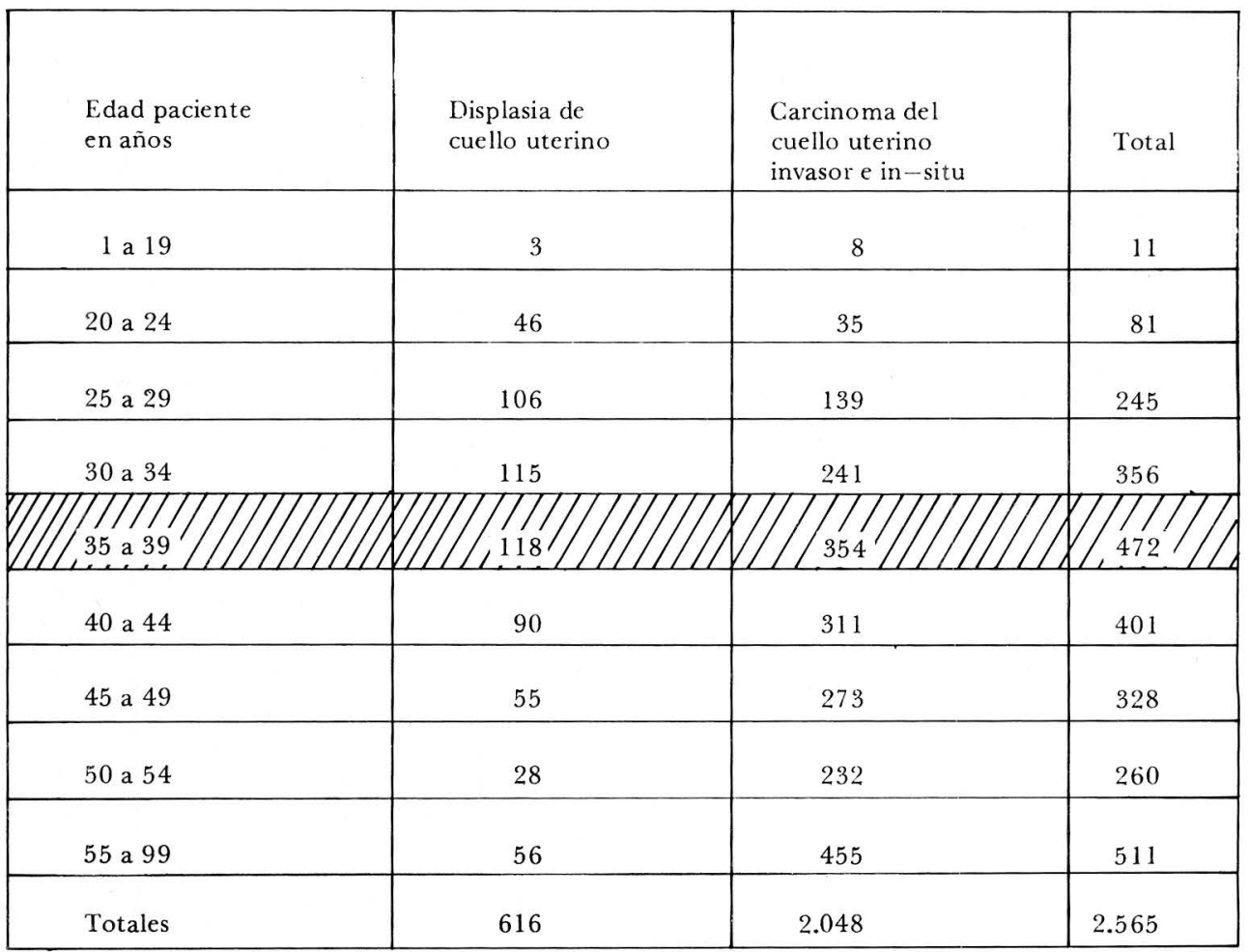


Cuadro No. 1A. Es descriptivo y permite ver como a 1957 pacientes o sea el 73.4\%/o se les tomó citología, no fué tomada o no se informó de ella en 709 casos o sea el $26.60 / 0$.

A 63 pacientes $(4.50 / 0)$ con estados avanzados III ó IV se les aplicó cobalto únicamente, como tratamiento paliativo.

La radioterapia combinada se utilizó en 989 pacientes con diferentes estados clínicos $(70.8 \mathrm{o} / \mathrm{o})$. Hubo 4 pacientes, o sea el $0.3 \mathrm{o} / \mathrm{o}$, en quienes debido a la resistencia al tratamiento inicial, este se completó con cirugía.

Cuadro No. 1B. Establece la diferencia que existe entre las pacientes de la zona urbana y las de la rural, ya que casi una cuarta parte de las que originaron información, procedían del campo y fueron enviadas a la Clínica Oncológica por los Centros de Salud del Departamento de Antioquia y sus áreas de influencia.

Cuadro No. 2. Se presentan en éste cuadro los grupos etáreos de iniciación de las relaciones sexuales y la localización de algunos tumores ginecológicos. Observamos como el $81.7 \% / 0$ de las pacientes con Carcinoma de cuello uterino, tuvieron su iniciación sexual antes de los 19 años. El porcentaje es similar en los carcinomas de vulva y vagina, órganos que tienen relación con el coito.El porcentaje para los carcinomas de endometrio y ovario fué de 58.7 o/o y68. $40 / 0$ respectivamente.

E1 gráfico No. 1 demuestra como es más frecuente el carcinoma de cuello en pacientes con iniciación sexual precoz a diferencia del carcinoma endometrial que aparece cuando la iniciación de las relaciones sexuales es más tardía. Después de los 30 años no se aprecia diferencia entre los dos carcinomas.

Gráfico No. 2. Muestra la frecuencia de las lesiones pre-malignas y malignas del cuello uterino, observándose que las displasias y el carcinoma in situ ocupan casi un $50 \% / 0$.

Gráfico No. 3. Representa la frecuencia de los estados clínicos del carcinoma invasor. El 57.4o/o corresponde al estado IIIb y le sigue el estado IIb.

\begin{tabular}{|lrc|}
\hline CUADRO No. $1-$ A & & \\
\hline & & \\
\hline \\
PACIENTES A QUIENES NO SE HIZO O INFORMO CITOLOGIA & 709 & $26.6 \%$ \\
\hline PACIENTES CON CITOLOGIA & 1.957 & $73.4 \%$ \\
\hline PACIENTES TRATADAS CON COBALTO SOLO & 66 & $4.5 \%$ \\
\hline PACIENTES TRATADAS CON COBALTO Y RADIUM & 989 & $70.8 \%$ \\
\hline PACIENTES TRATADAS CON COBALTO / RADIUM Y CIRUGIA & 4 & $0.3 \%$ \\
\hline
\end{tabular}

\begin{tabular}{|llll|}
\hline CUADRO No. $1-\mathrm{B}$ \\
\hline PACIENTES DE PROCEDENCIA RURAL & 641 & $24.5 \%$ \\
\hline PACIENTES DE PROCEDENCIA URBANA & 2.025 & $75.5 \%$ \\
\hline
\end{tabular}


CUADRO No. 2

\begin{tabular}{|c|c|c|c|c|c|c|c|c|c|c|c|c|}
\hline \multirow{2}{*}{$\begin{array}{c}\text { EDAD PRIMERA } \\
\text { RELACION } \\
\text { SEXUAL } \\
\end{array}$} & \multicolumn{10}{|c|}{ CARCINOMA DE : } & \multirow{2}{*}{$\begin{array}{l}\text { TOTAL } \\
\text { POR } \\
\text { EDAD }\end{array}$} & \multirow[b]{2}{*}{$\%$} \\
\hline & CUELLO & op & ENDOME. & $\%$ & VULVA & $\%$ & VAGINA & $\%$ & OVARIO & $0 \%$ & & \\
\hline-19 & 1.528 & 81.7 & 37 & 58.7 & 20 & 83.3 & 17 & 81.0 & 39 & 68.4 & 1.641 & 81.0 \\
\hline $20-24$ & 228 & 12.2 & 13 & 20.6 & 1 & 4.2 & 1 & 4.8 & 13 & 22.8 & 256 & 12.6 \\
\hline $25-29$ & 80 & 43.0 & 17 & 11.1 & 2 & 8.3 & 2 & 9.5 & 2 & 3.5 & 93 & 4.6 \\
\hline $30-34$ & 19 & 1.0 & 2 & 3.2 & 1 & 4.2 & 1 & 4.7 & 2 & 3.5 & 25 & 1.2 \\
\hline $35-39$ & 6 & 0.3 & 1 & 1.6 & 0 & - & 0 & - & 1 & 1.8 & 8 & 0.4 \\
\hline $40-44$ & 4 & 0.2 & 2 & 3.2 & o & - & 0 & - & $\theta$ & - & 6 & 0.3 \\
\hline $45-49$ & 1 & 0.1 & 1 & 1.6 & $\mathbf{0}$ & - & 0 & - & 0 & - & 2 & 0.1 \\
\hline $50-54$ & 1 & 0.1 & 0 & - & 0 & - & 0 & - & 0 & - & 1 & 0.0 \\
\hline $55-59$ & 3 & 0.2 & 0 & - & 0 & - & 0 & - & 0 & - & 3 & 0.1 \\
\hline TOTAL & 1.870 & 100 & 63 & 100 & 24 & 100 & 21 & 100 & 57 & 100 & 2.035 & 100.0 \\
\hline
\end{tabular}

GRA FICO No. 1

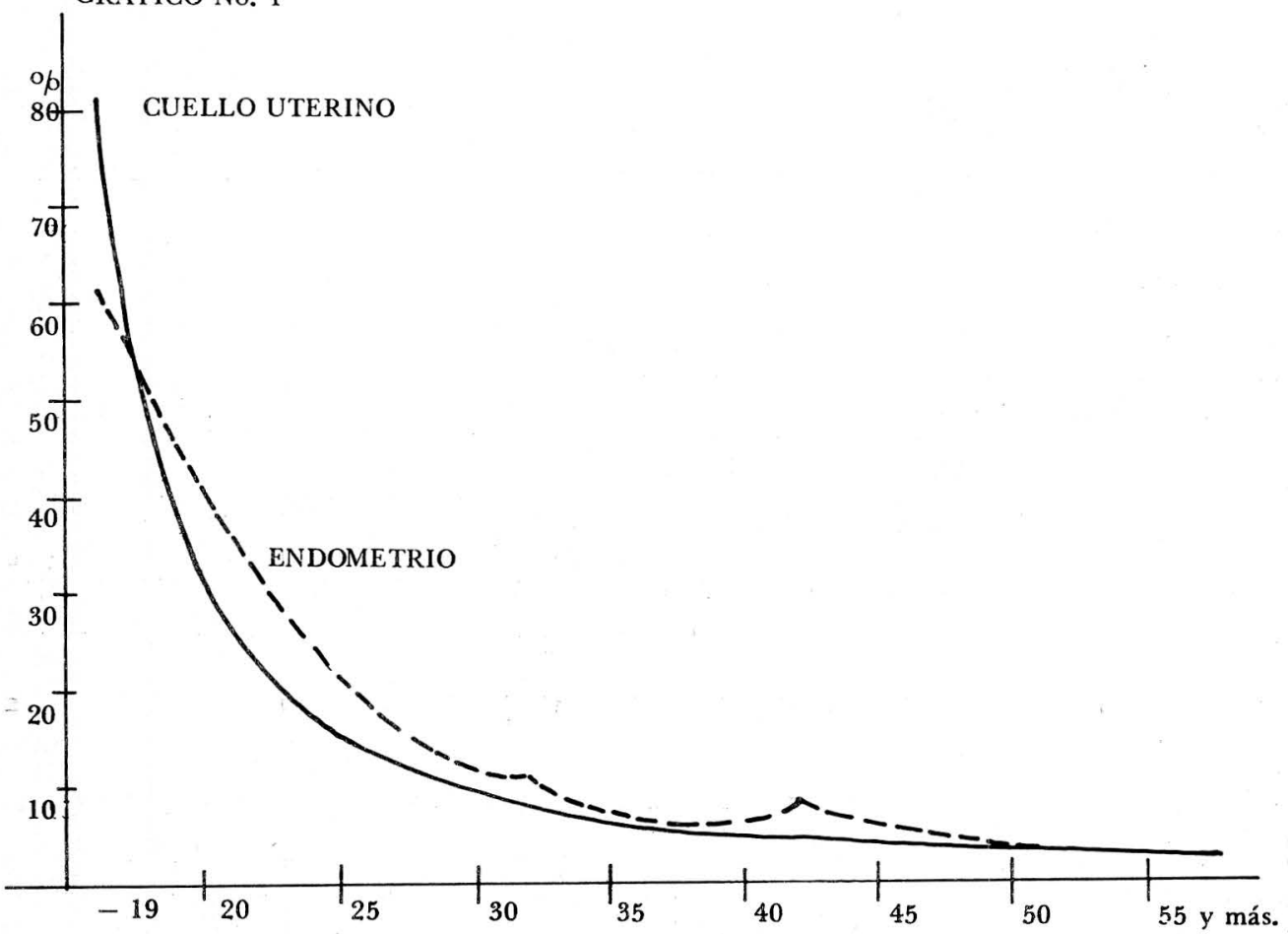




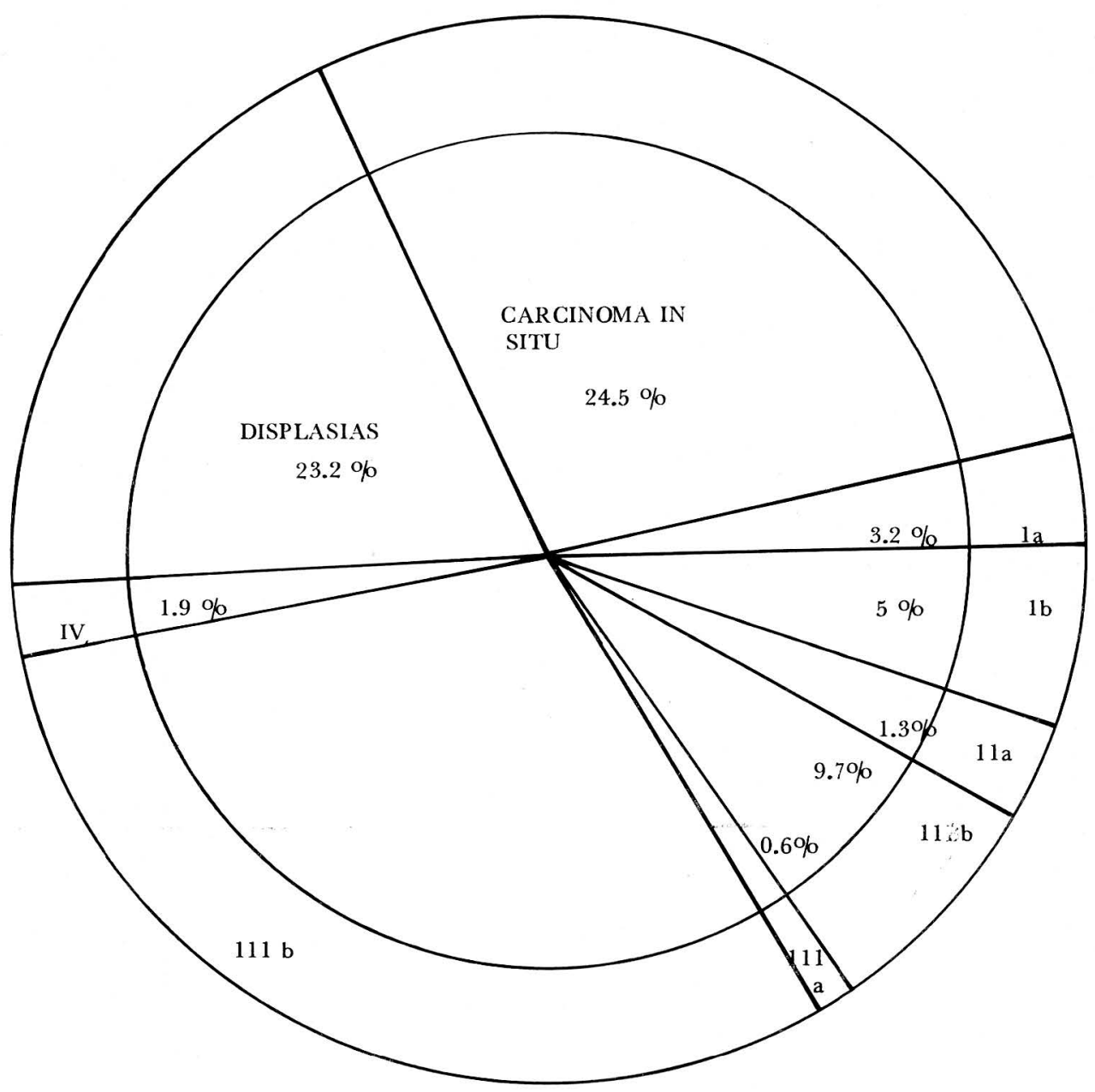

CUADRO No. 4

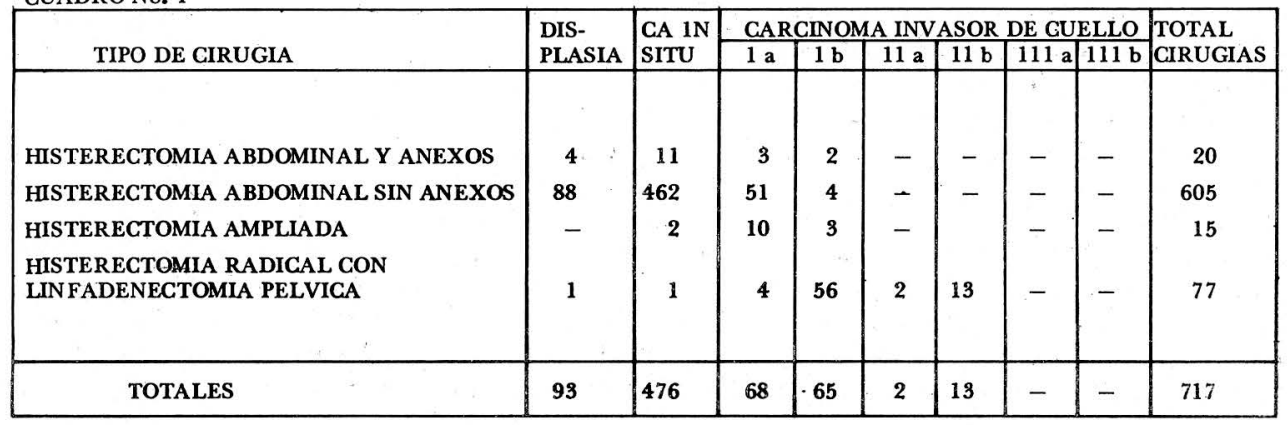




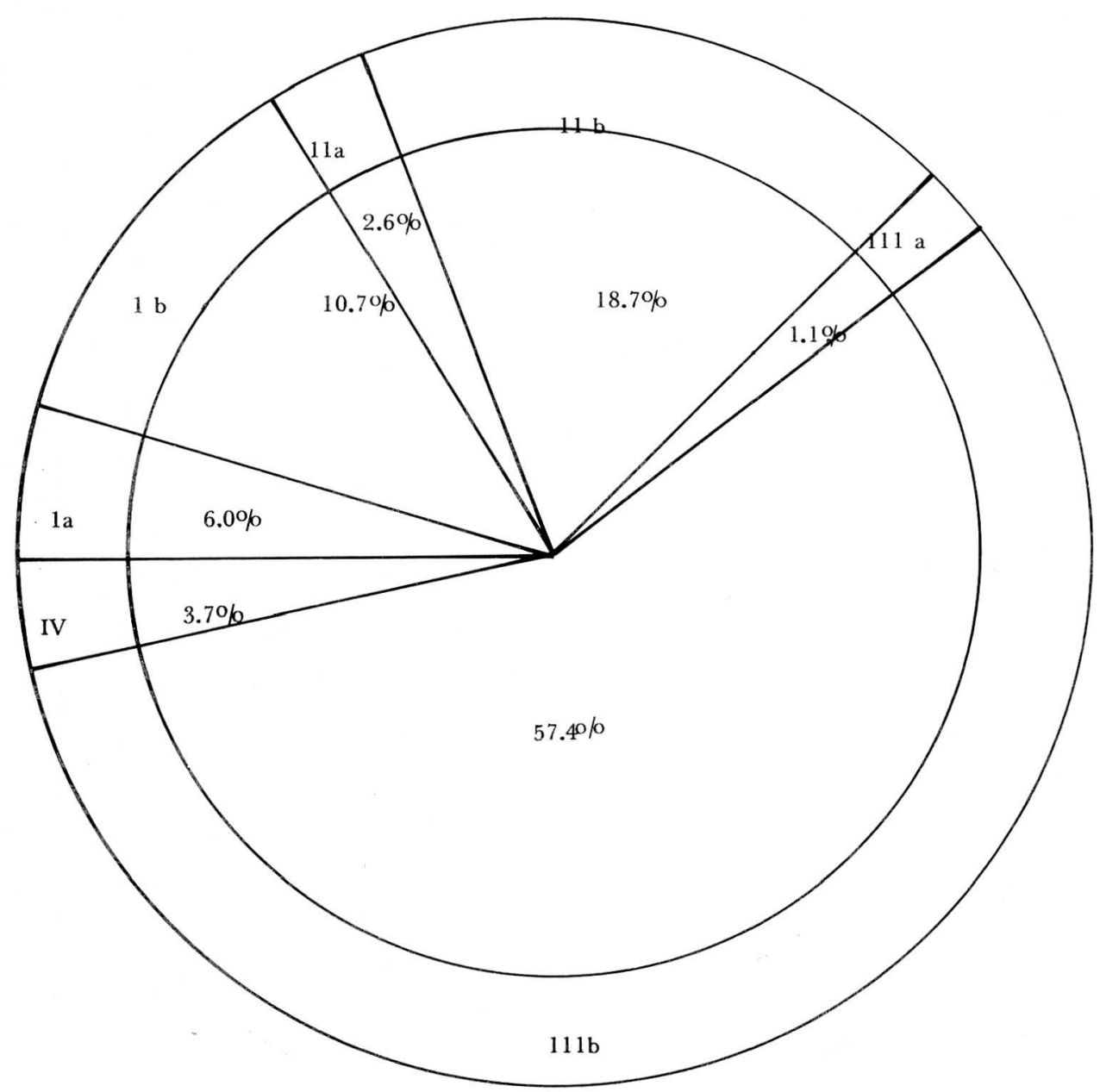

Cuadro No. 3. Nos llama la atención que el grupo etareo de 10 a 14 años, presente 208 casos que corresponden al $15 \% / o$, en 117 pacientes o sea el $8.5 \% / 0$ no se obtuvo información por haber muerto la mayoría antes de iniciar el trabajo.

Cuadro No. 4. Este cuadro coincide en general con las normas quirúrgicas internacionales para el tratamiento de las displasias y carcinomas del cuello uterino. Aparecen en él dos casos de histerectomía radical con linfadenectomía pélvica, uno en displasia y otro en carcinoma in situ. Esto se explica por el hecho de que el informe anatomopatológico solo reveló lesiones menores que las informadas en la biopsia previa.

Cuadro No. 5. Deben explicarse algunas cifras que llaman la atención. 65 pacientes con carcinoma de cuello estado Ib, quienes fueron tratadas con cobalto y radium, debido a que en una época se limitó la cirugía radical en el Departamento de Ginecología del Hospital San Vicente de Paul de Medellín y otras veces por considerar los casos, 
de alto riesgo quirúrgico. Tratados con cobalto únicamente 66 casos de carcinoma avanzado del cuello uterino, que solo eran susceptibles de tratamiento paliativo. Por circunstancias sociales y por la dificultad de permanecer largo tiempo en la ciudad se incluyeron en un protocolo especial.

De 887 pacientes que aparecen en control, pérdidas o tratadas en otros centros, la mayor parte de ellas son displasias que se encuentran en seguimiento en los centros de salud, clínica de oncología ginecológica del Hospital Universitario San Vicente de Paul e I.C.S.S.

\begin{tabular}{|c|c|c|c|}
\hline \multicolumn{2}{|c|}{ ED.SD } & NUMERO DIE P.ACILNTES & $\%$ \\
\hline 5 & -9 & 1 & 0.07 \\
\hline 10 & -. 14 & 208 & 14.95 \\
\hline 15 & -19 & 756 & 54.34 \\
\hline 20 & $-\quad 24$ & 224 & 16.10 \\
\hline 25 & -29 & 61 & 4.40 \\
\hline 30 & $\ldots 34$ & 17 & 1.22 \\
\hline 35 & -39 & 4 & 0.28 \\
\hline 40 & -44 & 3 & 0.21 \\
\hline & -49 & 0) & 0.00 \\
\hline
\end{tabular}

CUADRO No. 6

\begin{tabular}{|l|r|c|}
\hline \multicolumn{1}{|c|}{ TRATAMIENTO } & No. & $\%$ \\
\hline & & \\
SIN OTRO TRATAMIENTO & 2.618 & 98.19 \\
ELECTROCOAGULACION & 4 & 0.001 \\
LIGADURA DE HIPOGASTRIGAS & 7 & 0.003 \\
DERIVACION DE URETERES & 6 & 0.002 \\
HISTERECTOMIA POS RADIUM O COBALTO & 4 & 0.001 \\
COLOSTOMIA & 17 & 0.006 \\
VEJIGA ILEAL & 2 & 0.001 \\
CORRECION DE FISTULAS & 3 & 0.001 \\
OTROS & 5 & 0.002 \\
\hline
\end{tabular}

Cuadro No. 6 Observamos como de las 2.666 pacientes, 48 requieren procedimientos quirúrgicos adicionales tal como lo muestra el cuadro y fueron debidos a diversas complicaciones.

Cuadro No. 7. Representa la edad promedio encontrada entre nosotros para las pa- cientes con displasia, carcinoma in situ y cada uno de los estados clínicos. El promedio de edad para todos los carcinomas es de $43.50 / 0$.

Cuadro No. 8. Al correlacionar el resultado de la biopsia con el informe del material quirúrgico, vemos como de 306 casos de carcinoma in situ diagnosticados por la biopsia, no siempre múltiple como se exige en la actualidad, fueron confirmados 244. En 31 casos, el informe de estudio de la pieza quirúrgica fué de displasia en sus diferentes etapas y en igual número, 31 , las lesiones fueron mayores: carcinoma micro-invasor 21 , e invasores 10 . A éstos últimos se les complementó el tratamiento con cobalto.

Cuadro No. 9. Representa las entidades que enviaron pacientes con patología del cuello uterino. El primer lugar corresponde a los centros de Salud Departamentales y a sus áreas de influencia, con 625 casos o sea el 23.4o/o. Siguen en su orden el Municipio de Medellín y el Hospital San Vicente de Medellín.

Gráfico No. 4. En éste gráfico se muestra lo expuesto anteriormente. Caprecom y Profamilia han contribuído en forma positiva al diagnóstico precóz, como lo confirma el gráfico No. 6 .

Los 450 casos de carcinomas invasores provenientes del Departamento de Antioquia y su área de influencia están representados en el gráfico No. 5 y constituyen una dolorosa realidad del abandono en que se encuentran las zonas rurales en relación con las campañas para el diagnóstico oportuno por deficiencia de las Entidades encargadas de dar salud.

Cuadro No. 10. Presentamos la letalidad del carcinoma invasor del cuello uterino que destacamos fundamentalmente. Pero en el mismo cuadro podemos obtener la supervivencia si determinamos el complemento.

Ejemplo: en el grupo de 0 - 7 meses donde de 13 pacientes murieron 11 y sobrevivie- 
ron 2 , su letalidad es del $840 / 0$ y la supervivencia es su complemento o sea el 16o/o.

En cuanto al caso global según el gráfico No. 7, observamos un período comprendido entre los años 1972 a 1974 en el cual vemos una estabilización y luego en los años siguientes una caída bastante pronunciada y a partir del año 1976 permanece con una tasa de letalidad por debajo del $10 \mathrm{o} / \mathrm{o}$.

Nos llama la atención ésta caída de la letalidad con un enorme incremento de la supervivencia. Creemos que se ha logrado una mayor motivación de la población para consultar precozmente y en ésta forma lograr que los tratamientos sean más efectivos. Es de anotar que en el período de 8 15 meses de seguimiento, hubo un incremento en la letalidad hasta del $950 / 0$, probablemente debido a un inadecuado tratamien to por falta de equipos.

Cuadro No. 11. Representa el número de casos y las muertes en los diferentes estados clínicos del carcinoma. Como puede observarse, el estado IV dá el mayor porcentaje de letalidad con un 49 o/o siguiendo el estado III a con un 37.5\%/o y el IIIb con un 29.5o/o siendo el menor el del carcino. $\mathrm{ma}$ in situ con $0.7 \mathrm{o} / \mathrm{o}$, donde se presentó una muerte por causa diferente. La letalidad general fué de $16.5 \%$ o.

\section{Tablas de Vida:}

La No. 1. Nos muestra los carcinomas ginecológicos en general y se analiza en ésta tabla una cohorte de 2.255 pacientes de los 3.000 que comprende el estudio completo de casos pero que por no reunir éste grupo total los requisitos indispensables para la tabla de vida o no conocerse la fecha exacta del diagnóstico y observación, fué necesario excluír 795 pacientes.

De ésta cohorte cada 6 meses se eliminaban las muertes, los casos perdidos y aquellas pacientes que por las circunstancias no alcanzaban sino un determinado período de observación. En ésta tabla podemos observar que la esperanza de vida al iniciar el tratamiento era de 17.9 meses y que para

CUADRO NO. 5

\begin{tabular}{|c|c|c|c|c|c|c|c|c|c|c|}
\hline $\begin{array}{l}\text { COBALTO Y } \\
\text { RADIUM }\end{array}$ & DISPLASIAS & $\begin{array}{l}\text { CARCINO } \\
\text { IN SITU }\end{array}$ & $1 \mathrm{a}$ & $1 \mathrm{~b}$ & $11 \mathrm{a}$ & $11 \mathrm{~b}$ & $111 \mathrm{a}$ & $111 \mathrm{~b}$ & IV & TOTAL \\
\hline ERNST & & 1 & 2 & 65 & 23 & 202 & $\dot{8}$ & 635 & 12 & 948 \\
\hline OVOIDE. & & & 2 & 1 & 1 & 12 & 2 & 21 & & 39 \\
\hline RADIUM SOLO & & 4 & 2 & & & & & 3 & & 9 \\
\hline COBALTO SOLO & & & & & & & & 34 & 32 & 66 \\
\hline $\begin{array}{l}\text { TOTAL CON RADIO: } \\
\text { TERAPIA }\end{array}$ & & 5 & 6 & 66 & 24 & 214 & 10 & 693 & 44 & 1.062 \\
\hline INTERVENIDAS & 93 & 476 & 68 & 65 & 2 & 13 & 0 & 0 . & 0 & 717 \\
\hline TOTAL. & 617 & 654 & 84 & 149 & 35 & 259 & 16 & 801 & 51 & 2.666 \\
\hline $\begin{array}{l}\text { EN CONTROL, } \\
\text { PERDIDAS O TRA- } \\
\text { BAJADAS EN OTRO } \\
\text { CENTRO }\end{array}$ & 524 & 173 & 10 & 18 & 9 & 32 & 6 & 108 & 7 & 887 \\
\hline
\end{tabular}

CUADRO No. 7

\begin{tabular}{|l|c|c|c|c|c|c|c|c|c|c|}
\hline ESTADO & $\begin{array}{l}\text { DIS- } \\
\text { PLASIAS }\end{array}$ & $\begin{array}{l}\text { CA 1N } \\
\text { SITU }\end{array}$ & $1 \mathrm{~A}$ & $1 \mathrm{~B}$ & $11 \mathrm{~A}$ & $11 \mathrm{~B}$ & $11 \mathrm{~A}$ & $111 \mathrm{~B}$ & IV & TOTAL CASOS \\
\hline NUMERO DE PACIENTES & 617 & 654 & 84 & 149 & 35 & 259 & 16 & 801 & 51 & 2.666 \\
\hline EDAD PROMEDIO & 37.8 & 37.5 & 39.8 & 44.1 & 43.6 & 48.3 & 49.1 & 50.6 & 52.3 & $\begin{array}{c}\text { PROMEDIO } \\
\text { GENERAL } \\
43.5\end{array}$ \\
\hline
\end{tabular}


las personas que presentaban el mayor período de observación, 84 meses, su esperanza de vida era de 3 meses. Debemos anotar que el corte se hace sin haber muerto la última persona ya que estas continúan en observación en nuestro estudio.

Tabla No. 2. Se refiere al carcinoma invasor in situ y displasias. En éste caso tomamos una cohorte de 1939 pacientes con las cuales tenemos una esperanza de vida, a la iniciación del tratamiento, de 24.7 meses de vida. Esta cohorte se ha logrado llevar hasta 90 meses en el momento de pre- sentar este trabajo y su esperanza de vida es de 3 meses como el caso anterior.

Tabla No. 3. Para carcinoma invasor de cuello uterino.

En esta tabla se analiza una cohorte de 1.056 pacientes con una esperanza de vida de 23.8 meses al iniciar el tratamiento. Esta cohorte se ha logrado llevar también a 90 meses y la esperanza de vida en ese momento es de 3 meses.

(Ver gráficos 8 y 9 donde se aprecia la letalidad y pérdida de pacientes y la tendencia de acuerdo con los meses de seguimiento).

CUADRO No. 8

\begin{tabular}{|c|c|c|c|c|c|c|c|c|}
\hline \multirow{2}{*}{$\begin{array}{l}\text { RESULTADO A. P. } \\
\text { DEL UTERO }\end{array}$} & \multicolumn{7}{|c|}{ RESULTADO ANATOMOPATOLOGICO DE LA BIOPSIA INICIAL } & \multirow{2}{*}{$\begin{array}{l}\text { TOTAL } \\
\text { UTERO }\end{array}$} \\
\hline & $\begin{array}{l}\text { DISPLASIA } \\
\text { LIGERA }\end{array}$ & $\begin{array}{l}\text { DISPLASIA } \\
\text { MODERADA }\end{array}$ & $\begin{array}{l}\text { DISPLASIA } \\
\text { SEVERA }\end{array}$ & $\begin{array}{l}\text { CARCINOMA } \\
\text { IN SITU }\end{array}$ & $\begin{array}{l}\text { CARCINOMA } \\
\text { MICRO-INVA. }\end{array}$ & $\begin{array}{l}\text { CARCINOMA } \\
\text { INVASOR }\end{array}$ & $\begin{array}{l}\text { ADENO } \\
\text { CARCINOMA }\end{array}$ & \\
\hline DISPLASIA ÑIGE. & 2 & 1 & 4 & 9 & 1 & 2 & - & 19 \\
\hline DISPI.ASIA MOD. & 1 & 1 & 8 & 8 & 1 & 1 & - & 20 \\
\hline DISPLASIA SEV. & - & 2 & 28 & 14 & - & 2 & 1 & 47 \\
\hline $\begin{array}{l}\text { CARCINOMA IN } \\
\text { SITU }\end{array}$ & 3 & 12 & 41 & 244 & 11 & 13 & 1 & 325 \\
\hline $\begin{array}{l}\text { CARCINOMA MICRO } \\
\text { INVASOR }\end{array}$ & - & 1 & 3 & 21 & 12 & 5 & - & 42 \\
\hline $\begin{array}{l}\text { CARCINOMA } \\
\text { INVASOR }\end{array}$ & - & - & 2 & 10 & 5 & 83 & 6 & 106 \\
\hline ADENO CARCINOMA & - & - & - & - & - & 4 & & \\
\hline $\begin{array}{l}\text { TOTAL BIOPSIA } \\
\text { INICIAL }\end{array}$ & 6 & 17 & 86 & 306 & 30 & 110 & 39 & 594 \\
\hline
\end{tabular}

CUADKO No. 9

\begin{tabular}{|l|c|c|c|c|c|}
\hline ENTIDAD & DISPLASIAS & CA IN SITU & CA INVASOR & TOTAL & $\%$ \\
\hline & & & & & \\
CAPRECAN & 53 & 77 & 127 & 257 & 9.63 \\
PROFAMILIA & 39 & 96 & 79 & 214 & 8.02 \\
IC S S & 50 & 57 & 23 & 130 & 4.87 \\
H U S V de P & 56 & 69 & 267 & 392 & 14.70 \\
MUNICIPIO & 127 & 156 & 146 & 429 & 16.09 \\
DEPARTAMENTO & 56 & 119 & 450 & 625 & 23.44 \\
MEDICO PARTICULAR & 6 & 11 & 117 & 134 & 5.02 \\
CLINICA LUZ CASTRO & 6 & 31 & 37 & 74 & 2.77 \\
HOSPITAL P. T. U. & 4 & 4 & 54 & 62 & 2.32 \\
DESCONOCIDA & 220 & 34 & 95 & 349 & 13.09 \\
\hline TOTALES & 617 & 654 & 1.395 & 2.666 & 100.0 \\
\hline
\end{tabular}

CUADRO No. 11

\begin{tabular}{|l|c|r|c|}
\hline ESTADO CLINICO & MUERTES & CASOS & \% DE MUERTES \\
\hline \multicolumn{1}{|c|}{ CARCIONOMA IN SITU } & 1 & 654 & $*$ \\
$1 \mathrm{~A}$ & 2 & 84 & 2.4 \\
$1 \mathrm{~b}$ & 17 & 149 & 11.4 \\
$11 \mathrm{~A}$ & 4 & 35 & 11.4 \\
$11 \mathrm{~b}$ & 49 & 259 & 18.9 \\
$111 \mathrm{~A}$ & 6 & 16 & 37.5 \\
\hline $111 \mathrm{~b}$ & 236 & 801 & 29.5 \\
IV & 25 & 51 & 49,0 \\
\hline GLOBAL & 3.390 & 2.049 & 16.5 \\
\hline
\end{tabular}




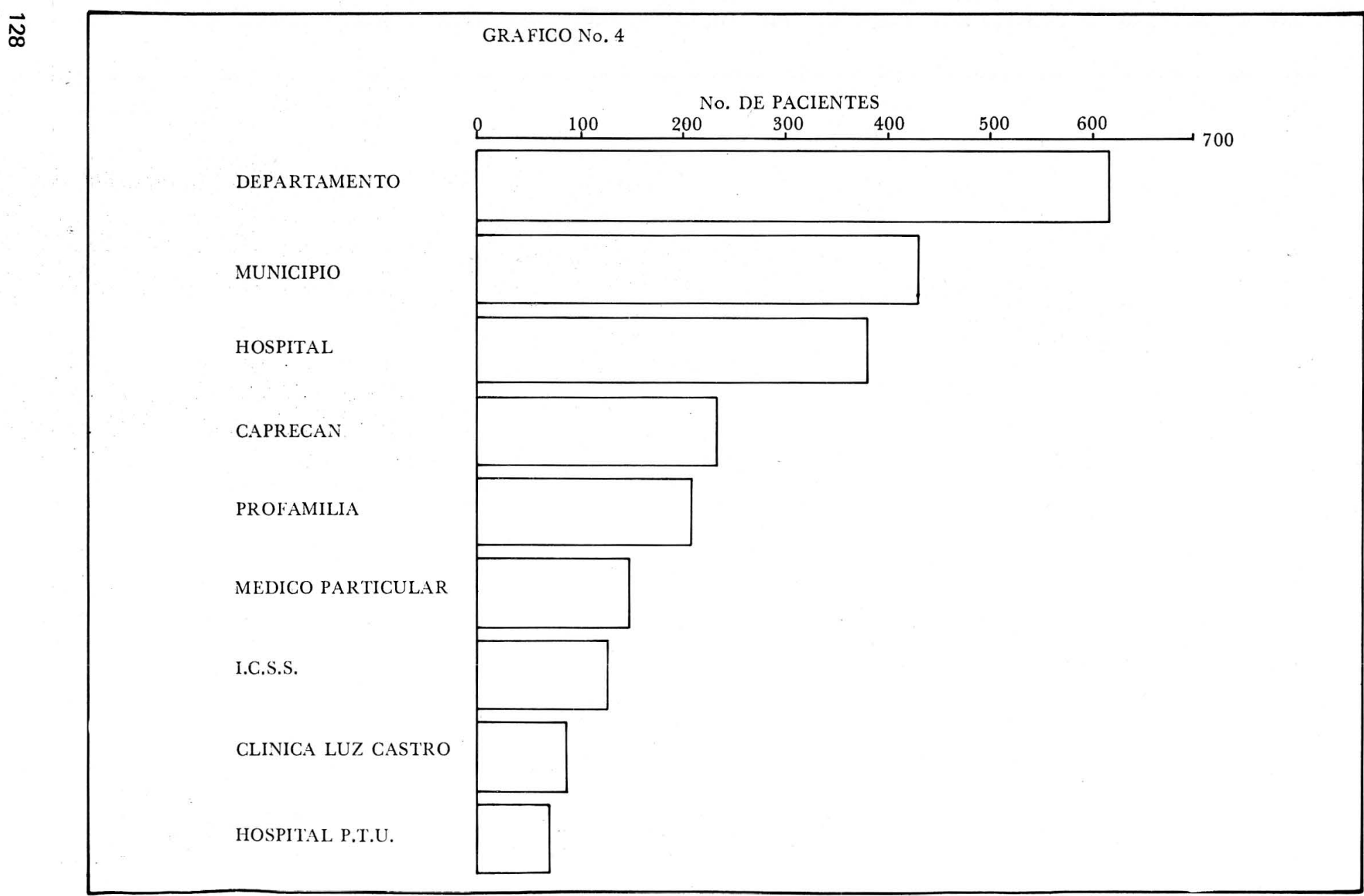


NUMERO DE PACIENTES

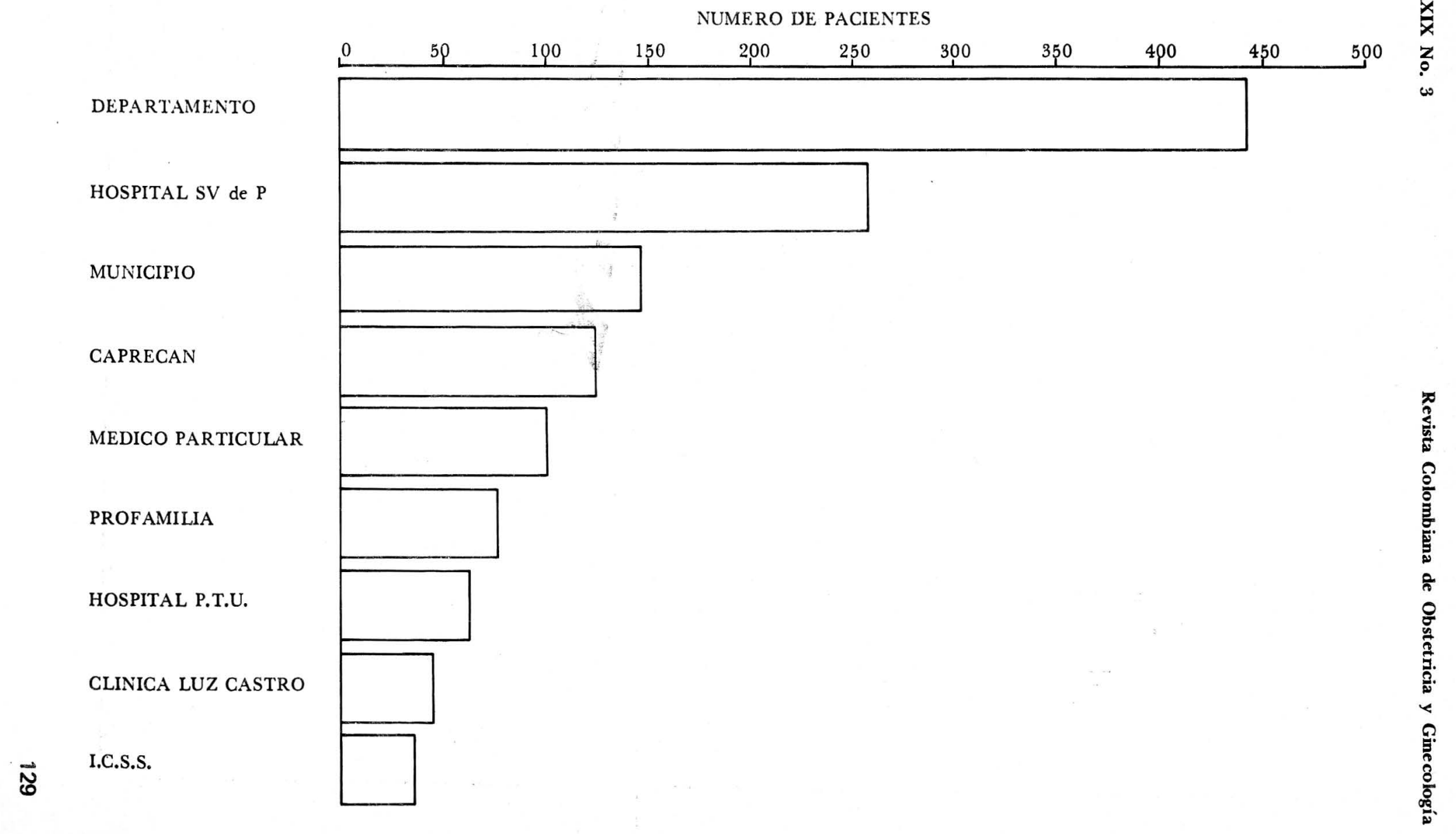


GRAFICO No. 6

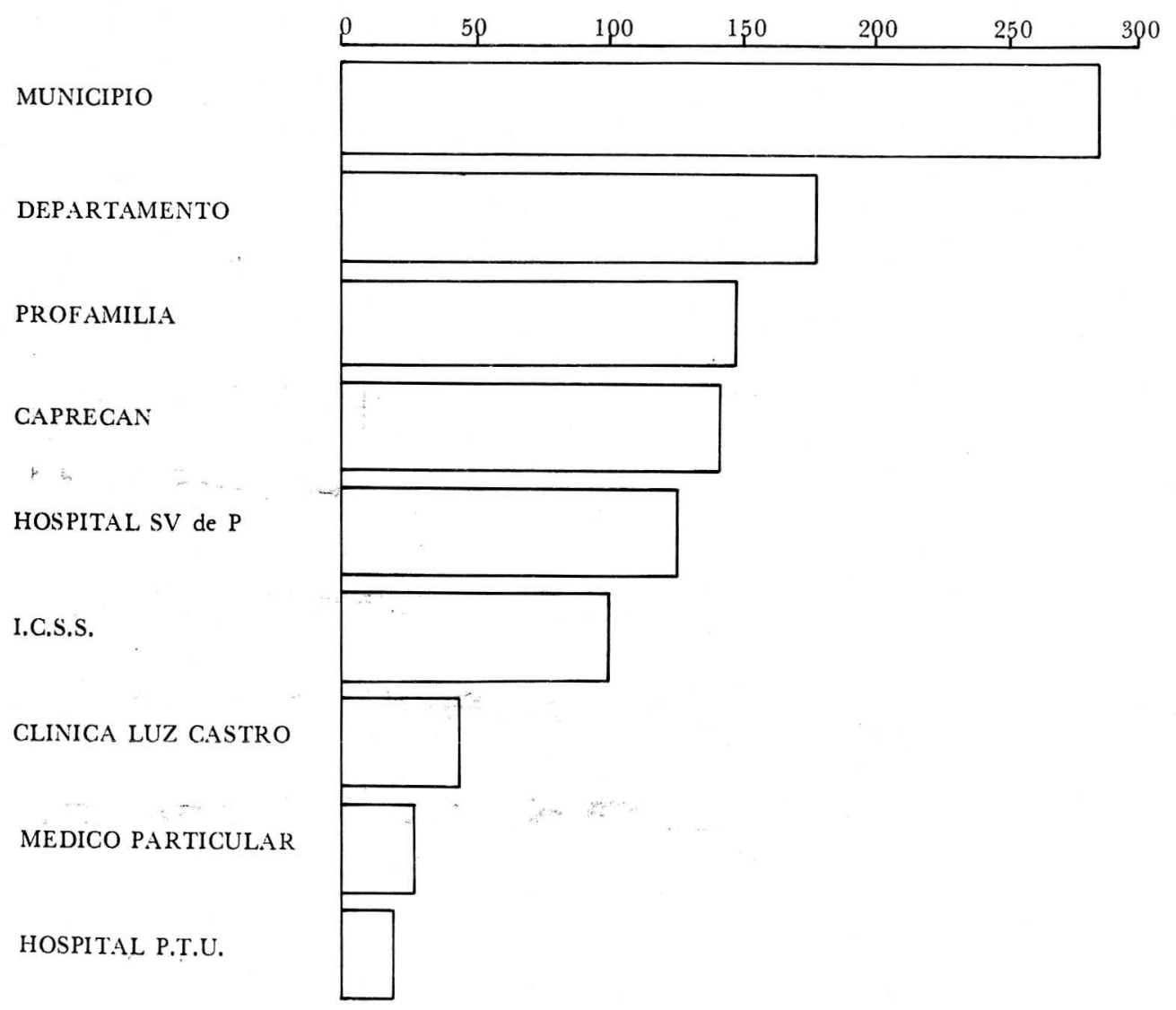


CUADRO No. 10

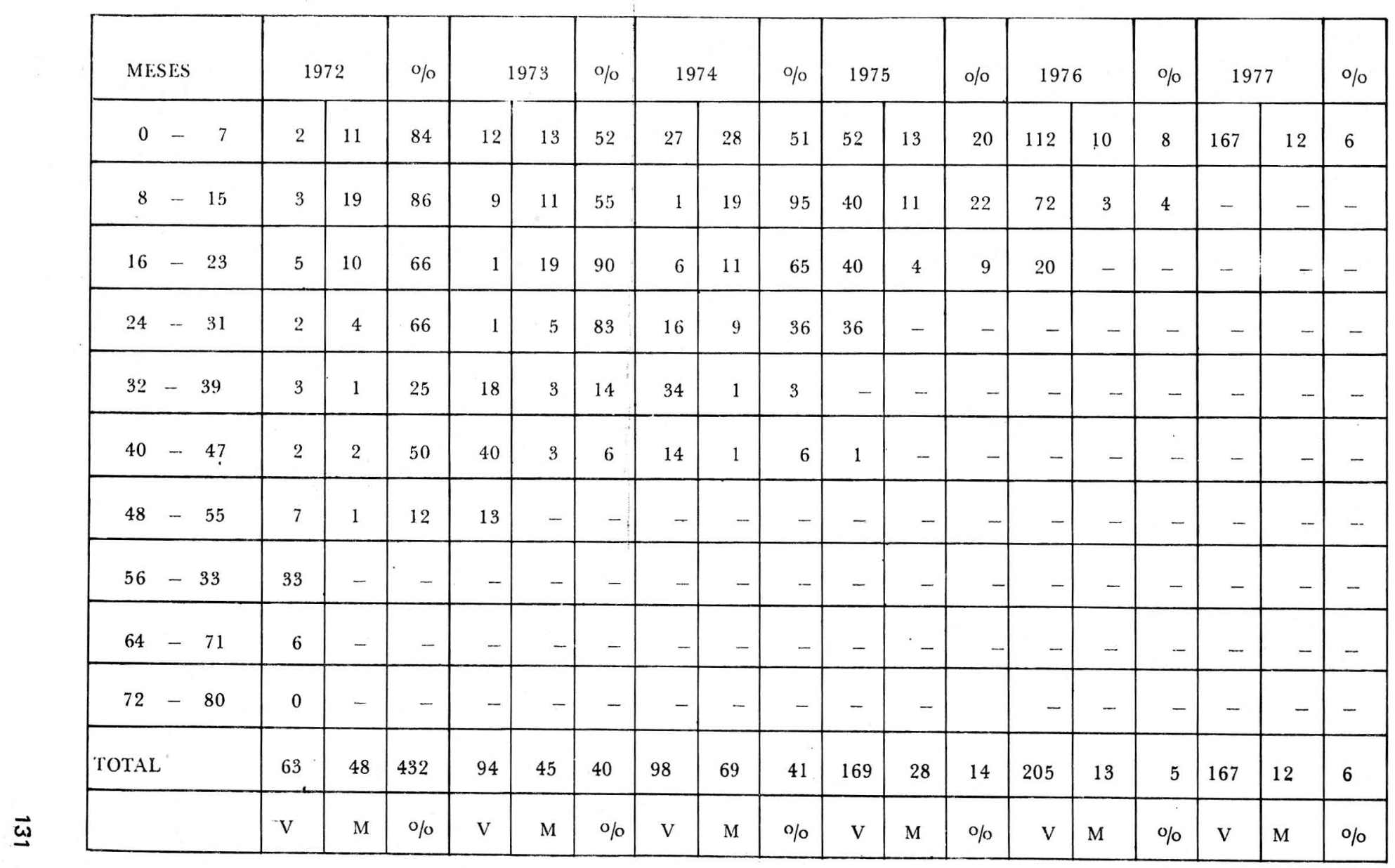









\begin{tabular}{|c|c|c|c|c|c|c|c|c|}
\hline $\mathrm{x}$ & I $\mathrm{X}$ & $\mathrm{AX}$ & $d x$ & $q x$ & W X & $L x$ & $\mathrm{~T} x$ & ex \\
\hline $0-6$ & 2.255 & 451 & 141 & 0.0656 & 54 & 11592 & 40.278 & 17.9 \\
\hline $7-12$ & 1.60 .9 & 399 & 153 & 0.1011 & 39 & 7881 & 28.686 & 17.8 \\
\hline $13-18$ & 1.018 & 160 & 16 & 0.0159 & 7 & 5559 & 20.805 & 20.4 \\
\hline $19-24$ & 835 & 165 & 44 & 0.0546 & 14 & 4.341 & 15.246 & 18.2 \\
\hline $25-30$ & 612 & 121 & 7 & 0.0116 & 6 & 3.270 & 10.905 & 17.8 \\
\hline $31-36$ & 478 & 139 & 16 & 0.0347 & 9 & 2.376 & 7.635 & 15.9 \\
\hline $37-42$ & 314 & 46 & 3 & 0.0096 & 1 & 1.736 & 5.259 & 16.7 \\
\hline $43-48$ & 264 & 71 & 2 & 0.0106 & 3 & 1.356 & 3.525 & 13.3 \\
\hline $\begin{array}{ll}49 & -54\end{array}$ & 188 & 44 & 0 & 0.0000 & 1 & 993 & 2.169 & 11.5 \\
\hline $55-60$ & 143 & 66 & 2 & 0.0148 & 2 & 648 & 1.175 & 8.2 \\
\hline $61-66$ & 73 & 28 & 1 & 0.0139 & 0 & 354 & 527 & 7.2 \\
\hline $67-72$ & 44 & 31 & 0 & 0.0000 & 2 & 82 & 175 & 4.0 \\
\hline $73-78$ & 11 & 1 & 0 & 0.0000 & 0 & 63 & 93 & 3.5 \\
\hline $79-84$ & 10 & 9 & 0 & 0.0000 & 1 & 30 & 30 & 3.0 \\
\hline & & & & & & & & \\
\hline
\end{tabular}




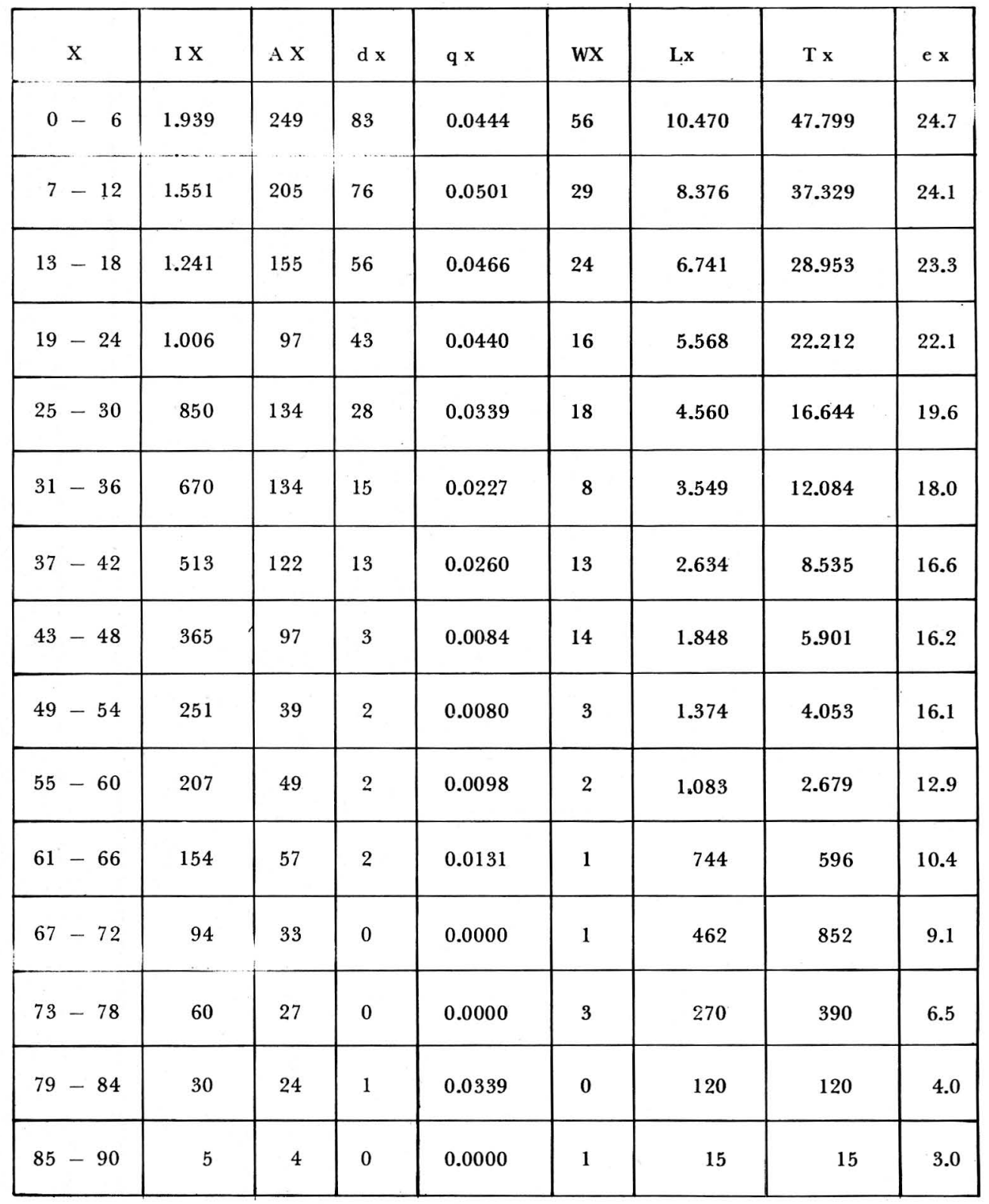




\begin{tabular}{|c|c|c|c|c|c|c|c|c|}
\hline $\mathrm{X}$ & I $x$ & $A X$ & $d x$ & $q x$ & $W x$ & $\mathrm{Lx}$ & $\mathrm{T} x$ & $\mathrm{e} x$ \\
\hline $0-6$ & 1.056 & 89 & 78 & 0.0767 & 27 & 5.754 & 25.152 & 23.8 \\
\hline $7-12$ & 862 & 112 & 74 & 0.0896 & 18 & 4.560 & 19.398 & 22.5 \\
\hline $13-18$ & 658 & 80 & 56 & 0.0888 & 13 & 3.501 & 14.838 & 22.6 \\
\hline $19-24$ & 509 & 47 & 42 & 0.0861 & 11 & 2.754 & 11.337 & 22.3 \\
\hline $25-30$ & 409 & 51 & 26 & 0.0650 & 12 & 2.187 & 8.583 & 21.0 \\
\hline $31-36$ & 320 & 42 & 14 & 0.0448 & 5 & 1.737 & 6.396 & 20.0 \\
\hline $37-42$ & 259 & 50 & 13 & 0.0528 & 3 & 1.356 & 4,659 & 18.0 \\
\hline $43-48$ & 193 & 43 & 3 & 0.0157 & 6 & 1.002 & 3.303 & 17.1 \\
\hline $49-54$ & 141 & 17 & 2 & 0.0143 & 2 & 783 & 2.301 & 16.3 \\
\hline $55-60$ & 120 & 28 & 2 & 0.0168 & 0 & 630 & 1.518 & 12.7 \\
\hline $61-66$ & 90 & 35 & 1 & 0.0112 & 1 & 429 & 888 & 9.9 \\
\hline $67-72$ & 53. & 20 & 0 & 0.0000 & 1 & 255 & 459 & 8.7 \\
\hline $73-78$ & 32 & 15 & 0 & 0.0000 & 2 & 141 & 204 & 6.4 \\
\hline $79-84$ & 15 & 11 & 1 & 0.0690 & 0 & 54 & 63 & 4.2 \\
\hline $85-90$ & 3 & 3 & 0 & 0.0000 & 0 & 9 & 9 & 3.0 \\
\hline
\end{tabular}


GRAFICO No. 8

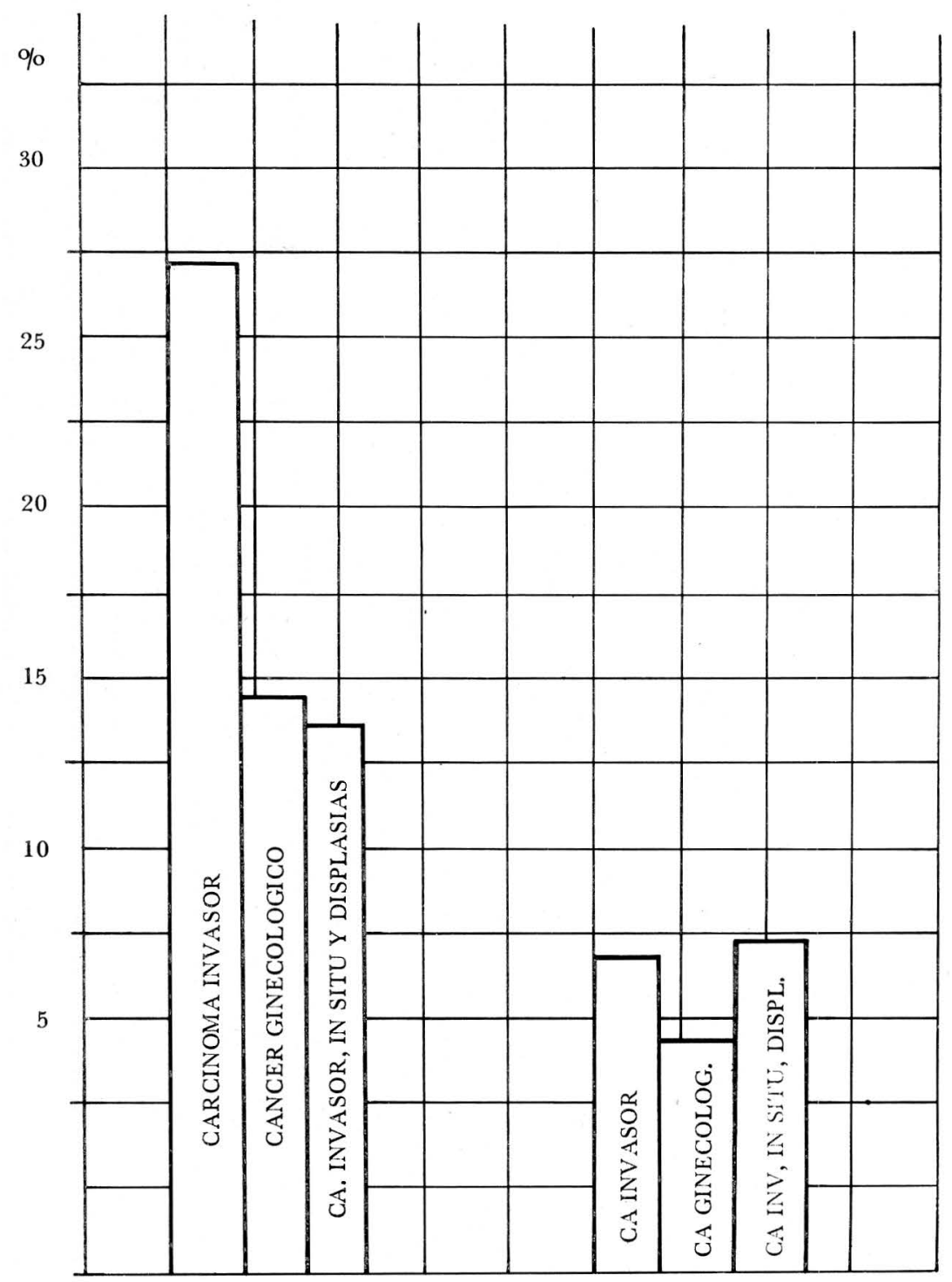


GRAFICO No. 9

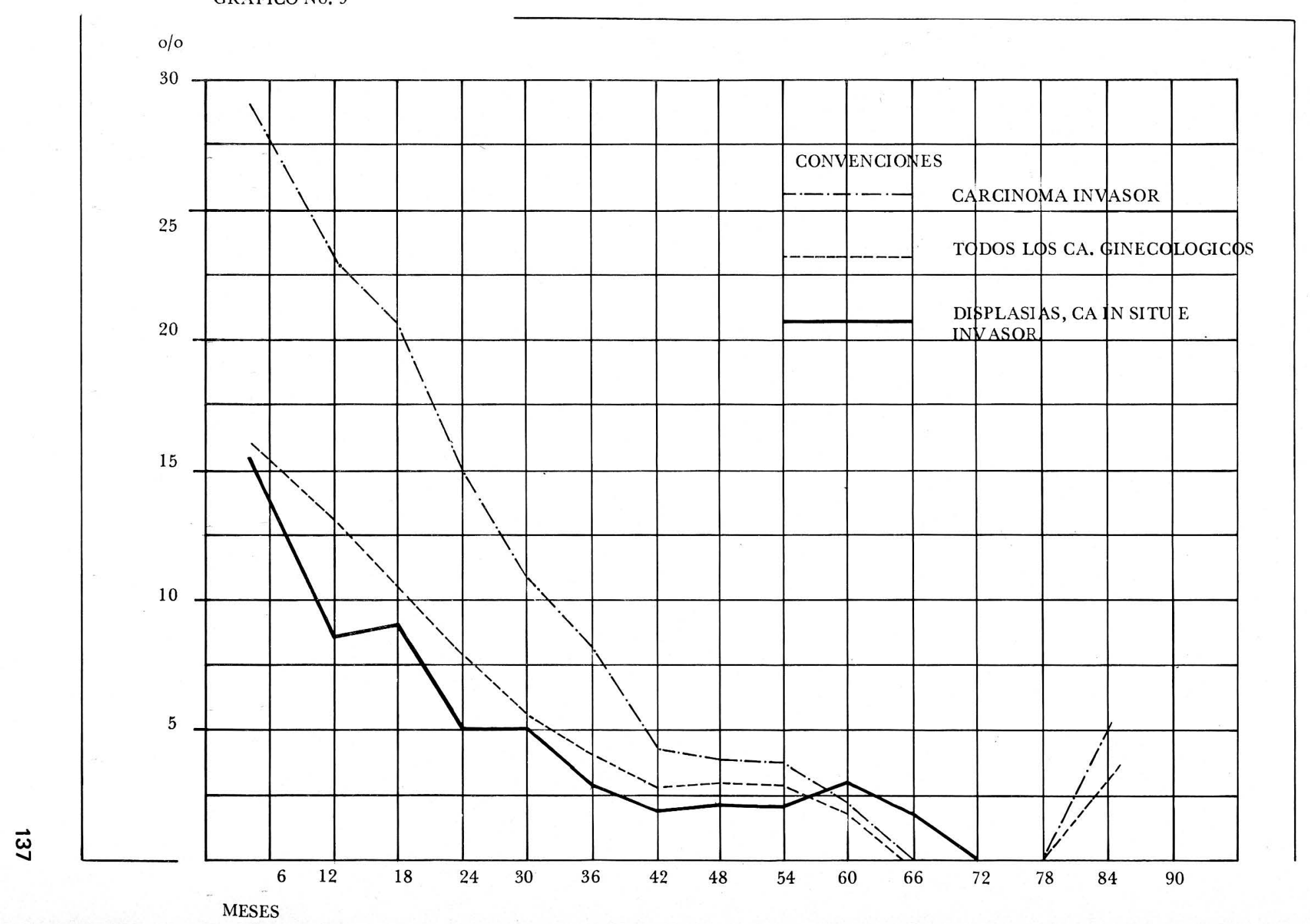




\section{RESUMEN Y CONCLUSIONES}

Después de un análisis de los datos recopilados podemos concluír:

1. En el grupo de 2.666 pacientes estudiadas durante un tiempo aproximado de 7 años, se destacó el grupo de edad comprendido entre 35 - 39 años como el de mayor frecuencia de lesiones del cuello uterino.

2. Se confirma en nuestro medio que las lesiones de cuello uterino son muy frecuentes en pacientes que iniciaron relaciones sexuales en edad temprana. 208 (15o/o) se presentaron en el grupo que las inició entre los 10 y 14 años.

3. El promedio de edad para las displasias y carcinoma in situ en el grupo de pacientes estudiadas fué de 37 años.

4. El carcinoma invasor ocupa el $51.3 \% / 0$ de las lesiones del cuello uterino y el estado IIIb el 57.4\%/o de los carcinomas invasores, lo cual demuestra la falta de mejores campañas para el diagnóstico temprano. La mayor parte de éstas pacientes provienen de la zona rural.

5. Se logró un seguimiento efectivo de más de $90 \%$ o con una pérdida aproximada del $10 \% / 0$ por diferentes causas, en un período de 7 años.

6. Se comprueba con éste estudio que con mística y dedicación se podrá llegar a controlar casi todas las pacientes estudiadas.

\section{SUMMARY AND CONCLUSIONS}

By analizing the date collected we can conclude :

1. That within the group of 2.666 patients studied during approx. 7 years, the age group between 35 to 39 was the one presenteng cervix lesions more frequently.

2. It is confirmed that in our envorinment, cervix lesions are very frequent in patients who started sex relations hips at an early age. $208(15 \% / 0)$ in the group, started them between 10 and 14 years old.

3. The average for displasias and carcinoma in situ was 37 years old in the group of patients under study.

4. Invasor carcinoma has $51.3 \mathrm{o} / \mathrm{o}$ of uterine cervix lesions and IIIb state the 57 . $40 / 0$ ofinvasor carcinomas facts which demostrate the lack of success ful campaigns for early diagnosis. A large aument of these patients come from rural areas.

5. We achieved more than $900 / 0$ of effective patients follow-up, with an approximate loss of $10 \% / 0$ dueto different causes, during the 7 years period.

6. It is proved with this study that with dedication almost of the studied patients may be followed-up. 